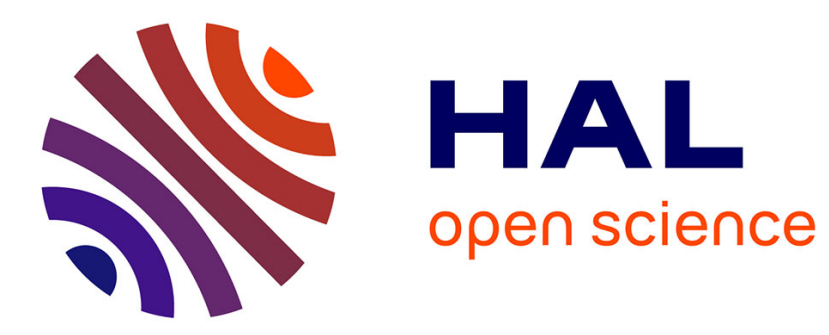

\title{
Cone fundamentals: A model for the future of colorimetry
}

Françoise Viénot

\section{To cite this version:}

Françoise Viénot. Cone fundamentals: A model for the future of colorimetry. International Journal of Lighting Research and Technology, 2016, 10.1177/1477153515624267 . hal-01565649

\section{HAL Id: hal-01565649 \\ https://hal.science/hal-01565649}

Submitted on 7 Sep 2017

HAL is a multi-disciplinary open access archive for the deposit and dissemination of scientific research documents, whether they are published or not. The documents may come from teaching and research institutions in France or abroad, or from public or private research centers.
L'archive ouverte pluridisciplinaire HAL, est destinée au dépôt et à la diffusion de documents scientifiques de niveau recherche, publiés ou non, émanant des établissements d'enseignement et de recherche français ou étrangers, des laboratoires publics ou privés. 


\title{
Cone fundamentals: A model for the future of colorimetry
}

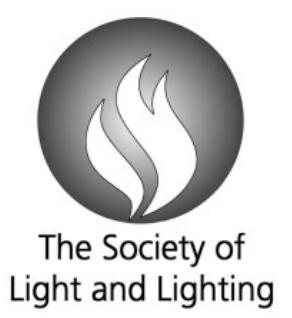

\author{
F Viénot PhD \\ Centre de Recherche sur la Conservation des Collections, \\ Muséum National d'Histoire Naturelle, Paris, France
}

Received 7 October 2015; Revised 25 November 2015; Accepted 1 December 2015

\begin{abstract}
Cone fundamentals is the name given to the relative spectral sensitivity of longwave sensitive, medium-wave sensitive and short-wave sensitive cones. Cone fundamentals represent the spectral absorptances of the retinal photopigments in the cones multiplied by the spectral transmittances of the ocular media through which light passes to reach the retina. The CIE 2006 cone fundamentals are based on the Stiles and Burch experimental colour matches and have been validated by recent microspectrophotometric measurements and modelling. A new physiologically based version of the MacLeod-Boynton chromaticity diagram is proposed by the $\mathrm{CIE}$ as well as a cone-fundamental-based $\left(x_{\mathrm{F}}, y_{\mathrm{F}}\right)$ chromaticity diagram. All the data necessary to use these concepts for high-precision colorimetric calculations are readily available.
\end{abstract}

\section{Introduction}

Since the introduction of the 1931 standard colorimetric observer, considerable knowledge on colour vision mechanisms has been acquired. Basing colorimetry directly on the physiological response of the cones rather than psychophysical measurements is a new approach to colour specification.

CIE Technical Committee 1-36 (TC 1-36) was established to propose chromaticity diagrams based on the best set of colourmatching functions and cone fundamentals available to date. Cone fundamentals is the name given to the relative spectral sensitivity of long-wave sensitive (L-), medium-wave sensitive (M-) and short-wave sensitive (S-) cones. As explicated by Judd, ${ }^{1}$ the cone fundamentals represent the spectral absorptances of the retinal photopigments included

Address for correspondence: F Viénot, Centre de Recherche sur la Conservation des Collections (CRCC, USR3224), Muséum National d'Histoire Naturelle, 36 rue Geoffroy Saint-Hilaire, Paris 75005, France.

E-mail: vienot@mnhn.fr in the cones multiplied by the spectral transmittances of the ocular media through which light passes to reach the retina. The CIE $2006^{2,3}$ cone fundamentals are based on the Stiles and Burch $^{4}$ experimental colour matches and have been validated by recent microspectrophotometric measurements and modelling. A new version of the MacLeodBoynton chromaticity diagram based on cone fundamentals is being proposed, ${ }^{5}$ as well as a cone-fundamental-based $\left(x_{\mathrm{F}}, y_{\mathrm{F}}\right)$ chromaticity diagram. All the data are now fixed. Tables are available in the CIE publications. ${ }^{2}$ Here, I present the CIE 2006 cone fundamentals, explaining how they have been derived and how to use them for colorimetry.

\section{Deriving the cone fundamentals}

The primary goal of TC 1-36 was to propose cone fundamentals that are securely grounded on colour-matching experiments with real observers and that would comply with the known physiology of the colour vision system. 


\section{F Viénot}

A comprehensive set of colour-matching data has been collected by Stiles and Burch ${ }^{4}$ from 47 colour normal observers. The colour matches were obtained at clearly photopic luminances, on a $10^{\circ}$ field, using spectral red, green and blue primaries expressed in energy units. The Stiles-Burch colour matching data are recognised as the most secure experimental basis for deriving cone fundamentals.

Independently, a physiological approach has allowed Stockman and Sharpe $e^{3,6,7}$ to derive estimates of the cone spectral sensitivities for a $2^{\circ}$ viewing angle. Given the fact that dichromats lack one family of cone pigment, and that S-cone responses can be cancelled by appropriate chromatic adaptation, these authors have been able, using heterochromatic flicker photometry, to isolate and directly measure the L- and M-cone spectral sensitivities. In parallel, the gene sequences associated with both L-cone and M-cone photopigments were analysed in order to associate each spectral sensitivity with a known genotype. Such information has made it possible to associate each spectral sensitivity with the identified normal genotype of the examined dichromat. The S-cone spectral sensitivity has been isolated in blue-cone monochromats and in chromatically adapted colour normal observers.

At this stage, an intermediate calculation step was introduced by Stockman

and Sharpe. ${ }^{6}$ In 1955, a small (interim) set of colour matches had been collected by Stiles and Burch $^{8}$ on a $2^{\circ}$ field which could be compared with the well-characterized cone spectral sensitivities for a $2^{\circ}$ viewing angle. Provided the individual dichromatic data were adjusted for the macular pigment and the lens density, a reasonable linear fit between the data sets could be found, thus validating the spectral sensitivity for every cone family.

To reinforce the validity of the cone fundamentals, a linear transformation between the $10^{\circ}$ cone spectral sensitivities and the $10^{\circ}$ StilesBurch colour-matching functions had to be demonstrated. Differences between colour assessments on a $10^{\circ}$ field and colour assessments on a $2^{\circ}$ field originate from differences in macular pigmentation and in photopigment absorption. Given some adjustment in lens optical density, in macular pigment optical density and in photopigment optical density, the best fit was found between the previously derived cone spectral sensitivities and the $10^{\circ}$ Stiles and Burch observers' colour matching functions.

Ultimately, CIE TC 1-36 adopted the cone fundamentals $\bar{l}_{10}(\lambda), \bar{m}_{10}(\lambda), \bar{s}_{10}(\lambda)$ for a $10^{\circ}$ viewing angle derived from the $10^{\circ}$ colour-matching functions in the experimental red, green and blue colour space (Figure 1).
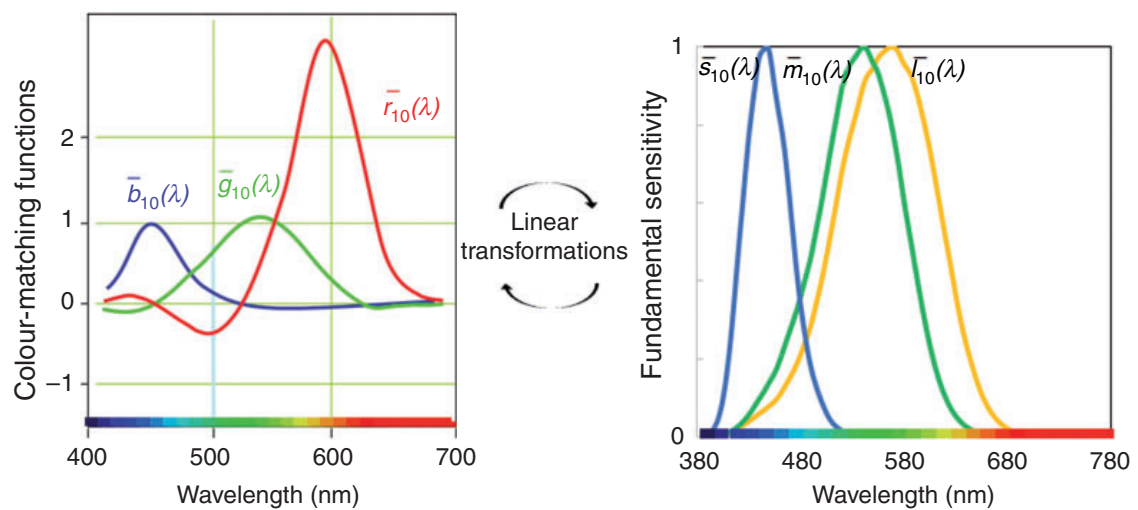

Figure 1. $10^{\circ}$ Stiles and Burch observers' colour matching functions and $10^{\circ}$ cone fundamentals. 


\section{The CIE 2006 cone fundamental framework}

Although the above described process had allowed CIE TC 1-36 to achieve its primary goal of establishing $10^{\circ}$ cone fundamentals, it also enabled the committee to propose a cone fundamental model incorporating ocular media, macular pigment and photopigment density parameters. The model consists of a framework that can be applied in both directions.

Here, the approach relies on the fact that the colour match is initiated by the absorption of photons in the cones, although it is measured in the corneal plane. By correcting the $10^{\circ}$ cone fundamentals for the absorption of the ocular media and the macular pigment, and taking into account the optical densities of the cone visual pigments, the spectral absorbance function of the dilute cone pigment can be modelled. Conversely, the $\bar{l}(\lambda), \bar{m}(\lambda), \bar{s}(\lambda)$ cone fundamentals for a $2^{\circ}$ viewing field can be reconstructed, by incorporating into the fundamental framework the $2^{\circ}$ values of the parameters. Thus, for a colour stimulus $\varphi(\lambda)$, the $2^{\circ}$ fundamental values are given as

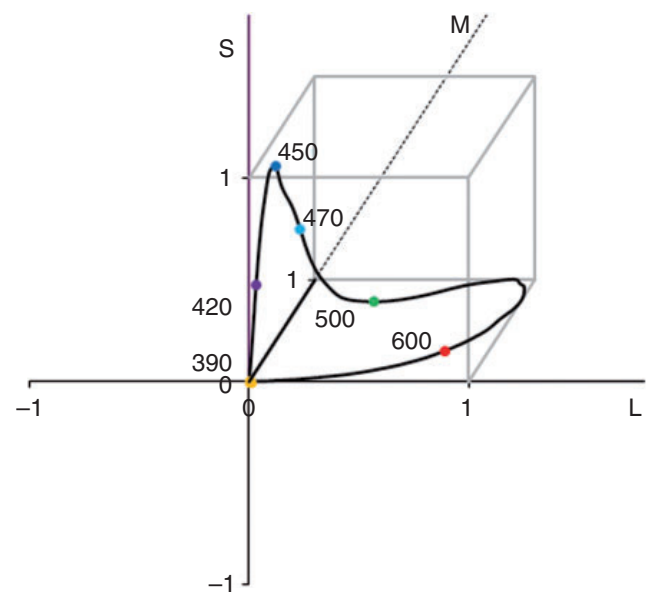

Figure 2. Representation of the monochromatic stimuli in the LMS cone fundamental space. The cone fundamentals are normalised to unit-peak values. The selected wavelengths are given in $\mathrm{nm}$.

$$
\begin{aligned}
L & =\int \varphi(\lambda) \bar{l}(\lambda) \mathrm{d} \lambda \\
M & =\int \varphi(\lambda) \bar{m}(\lambda) \mathrm{d} \lambda \\
S & =\int \varphi(\lambda) \bar{s}(\lambda) \mathrm{d} \lambda
\end{aligned}
$$

Figure 2 shows the monochromatic stimuli in the LMS cone fundamental space.

The diluted photopigment absorbance spectrum $A(\lambda)$ is really the core of the framework (Figure 3). Not only has the return path made it possible to construct the cone fundamentals for the $2^{\circ}$ visual field, it also makes it possible to construct the cone fundamentals for any field size.

Because colour vision varies with the diameter of the viewing field and with the age of the observer, the fundamental model allows the user to reconstruct cone fundamentals and adjust colour specification over a continuous range of field diameters from $1^{\circ}$ to $10^{\circ}$ and as a function of age. Thus, parameters along the reverse pathway are left to the researcher's choice: Specifically, setting the peak wavelength of the receptor photopigment, modifying the optical density of the photopigment in the receptor which changes the width of the effective photopigment optical density and adjusting the optical density of the macular pigment and the optical density of the lens.

\section{From colorimetry to photometry}

CIE TC 1-36 could have stopped work with the definition of cone fundamentals. Indeed, these definitions form the content of CIE publication 170-1:2006. ${ }^{2,9}$ However, further progress has been made resulting in CIE publication 170-2 (in press).

On the one hand, based on the modern physiological understanding that luminance arises from the sum of $\mathrm{L}$ - and M-cone responses, a new cone-fundamental-based spectral luminous efficiency function $V_{\mathrm{F}}(\lambda)$ 


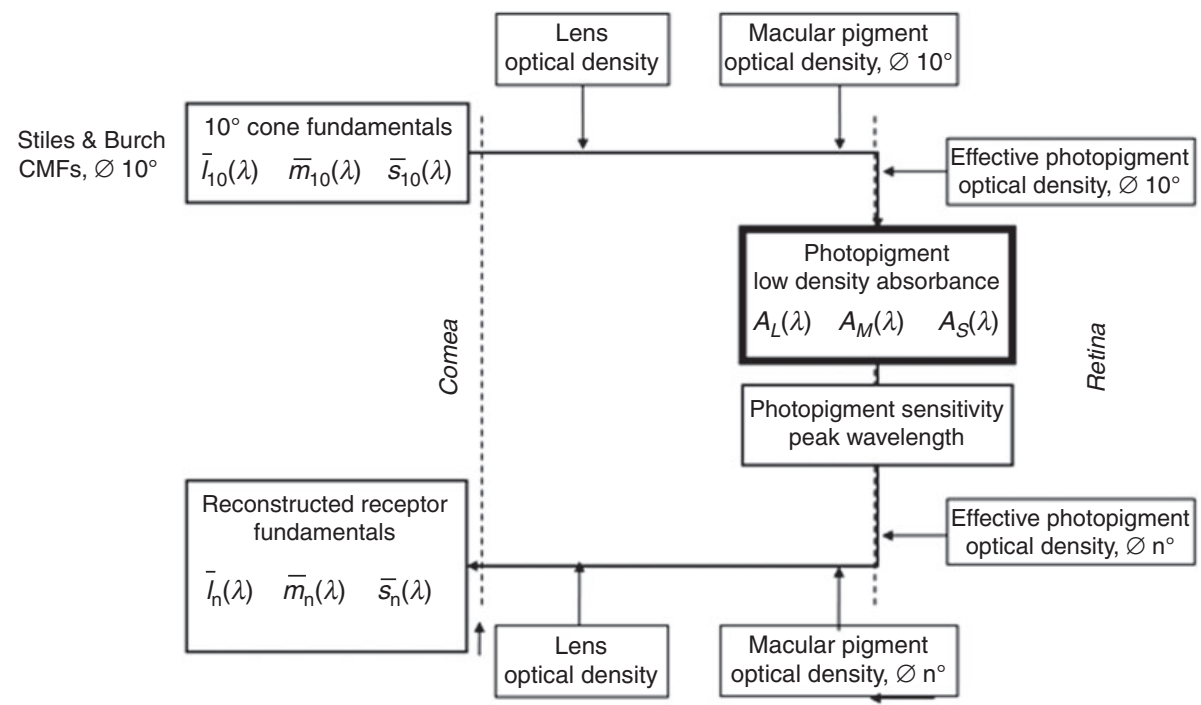

Figure 3. The CIE 2006 cone fundamental framework for reconstructing cone fundamentals, for any viewing field.

has been defined in terms of the linear combination of the $\mathrm{L}-$ and M-cone fundamentals.

$$
V_{\mathrm{F}}(\lambda)=k_{\mathrm{L}} \bar{l}(\lambda)+k_{\mathrm{M}} \bar{m}(\lambda)
$$

The spectral luminous efficiency function $V(\lambda)$ of the 1924 standard photometric observer has been known to markedly underestimate the effect of short wavelengths which has lead the CIE to propose an improved spectral luminous efficiency function $V_{M}(\lambda)$ in $1990 .{ }^{10}$ Later, new heterochromatic flicker photometry measurements in colour normal observers, associated with the identification of the L-cone pigment genotype, have allowed Sharpe et al..$^{11-13}$ to verify that a linear transformation of the Lcone and the M-cone fundamentals could be adjusted to match $V_{\mathrm{M}}(\lambda)$ provided suitable weights were applied (Figure 4).

\section{The MacLeod-Boynton chromaticity diagram}

Once photometry is associated with colorimetry, it is possible to propose a chromaticity diagram that lies in a constant LM-luminance plane. In the LMS cone fundamental space, the constant LM-luminance plane is parallel to the $\mathrm{S}$-axis because the $\mathrm{S}$-cone response does not contribute to luminance. ${ }^{5}$ The plane is oriented so that the total contributions of the L-cone and the M-cone responses to the luminance response remain constant within the surface.

In the so-called MacLeod-Boynton chromaticity diagram, the abscissa $l$ shows the fraction of luminance attributable to L-cones; the ordinate $s$ shows the S-cone response relative to luminance, scaled for convenience to fit within the $[0,1]$ range (Figures 5 and 6).

$$
\begin{aligned}
& l_{\mathrm{MB}}=\frac{L_{\mathrm{MB}}}{L_{\mathrm{MB}}+M_{\mathrm{MB}}} \\
& s_{\mathrm{MB}}=\frac{S_{\mathrm{MB}}}{L_{\mathrm{MB}}+M_{\mathrm{MB}}}
\end{aligned}
$$

Hence, the MacLeod-Boynton tristimulus values $L_{\mathrm{MB}}, M_{\mathrm{MB}}$ and $S_{\mathrm{MB}}$ can be calculated by integrating the product of the stimulus function and the cone fundamentals, applying appropriate weights to the cone fundamentals, with respect to LM-luminance and to $s$ scaling in the MacLeod-Boynton chromaticity diagram. 


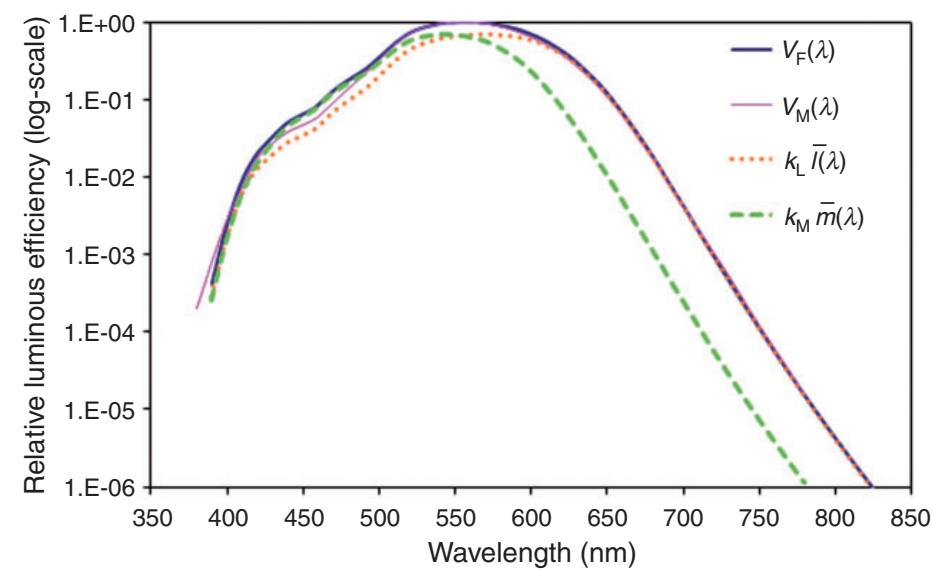

Figure 4. The $2^{\circ}$ cone-fundamental-based spectral luminous efficiency function $V_{F}(\lambda)$ defined as the linear combination of the cone fundamentals $\bar{l}(\lambda)$ and $\bar{m}(\lambda)$, compared with the CIE 1988 modified spectral luminous efficiency function $V_{\mathrm{M}}(\lambda)$ (log scale). Coefficients $k_{\mathrm{L}}$ and $k_{\mathrm{M}}$ in the legend refer to equation 2 .
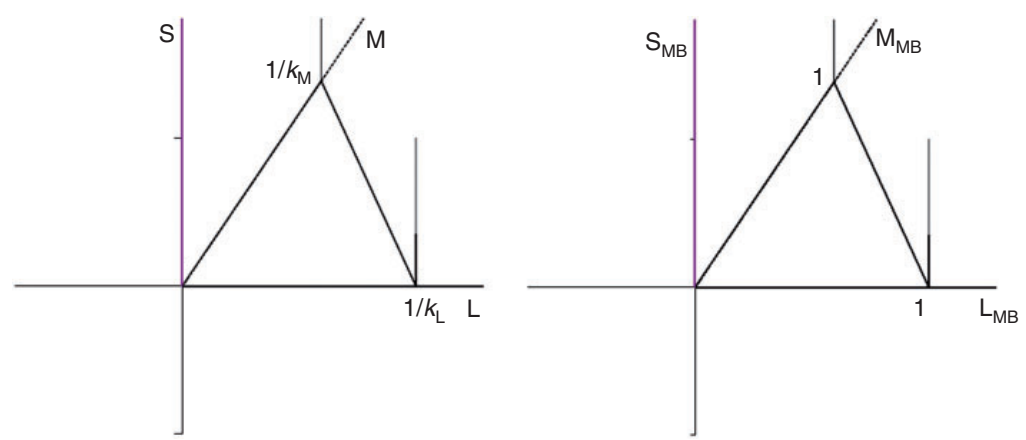

Figure 5. Left: Representation of the constant LM-luminance plane in the cone fundamental space. Right: the axes of the cone fundamental space are appropriately re-scaled to conform to the MacLeod-Boynton representation.

In the LMS tristimulus space, the alychne plane that represents the locus of colour stimuli of zero LM-luminance includes the S-cone fundamental axis (Figure 7).

\section{Cone-fundamental-based chromaticity diagram and tristimulus values}

On the other hand, in order to comply with industrial practice and the current CIE practice, the final goal consists of offering colorimetric tools in the form of a traditional $X Y Z$ specification including a $x, y$-like chromaticity diagram.

The cone-fundamental-based spectral tristimulus values $\bar{x}_{\mathrm{F}}(\lambda), \bar{y}_{\mathrm{F}}(\lambda)$ and $\bar{z}_{\mathrm{F}}(\lambda)$ are obtained from a linear transformation of the cone fundamentals. The principles that guided the development of the current CIE standard colorimetric system were followed ${ }^{14}$ when defining the new $\left(x_{\mathrm{F}}, y_{\mathrm{F}}\right)$ cone-fundamental-based chromaticity diagram. 


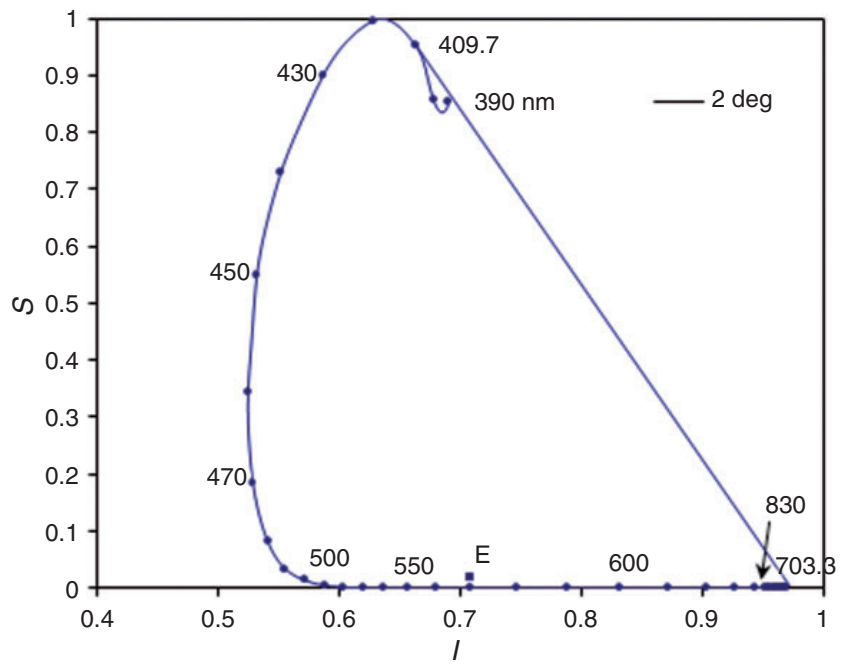

Figure 6. The MacLeod-Boynton chromaticity diagram, for the $2^{\circ}$ observer.

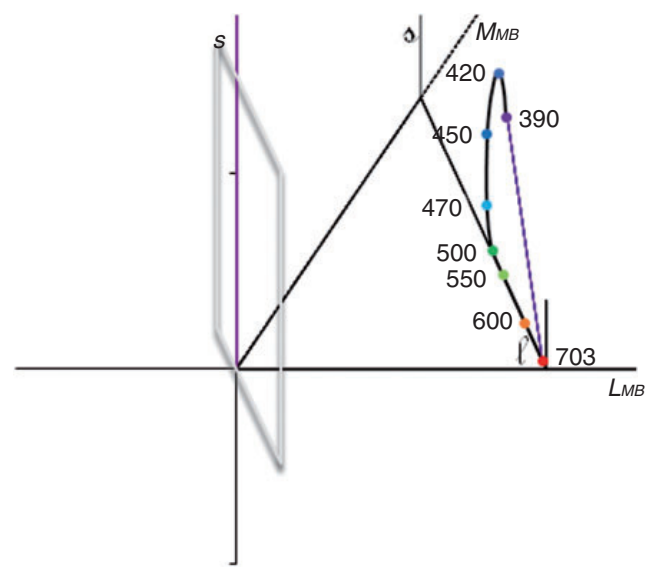

Figure 7. Representation of the MacLeod-Boynton chromaticity diagram in the constant LM-luminance plane. The axes of the cone fundamental space are appropriately re-scaled. The selected wavelengths are given in $\mathrm{nm}$. A blurred contour is used to sketch the alychne plane.

1) All cone-fundamental-based tristimulus values of real colours are non-negative (Figure 8).

2) The cone-fundamental-based tristimulus value $Y$, alone, yields the luminance. Thus, the $\mathbf{X}_{\mathrm{F}}$ and $\mathbf{Z}_{\mathrm{F}}$ primaries are located in the alychne plane.

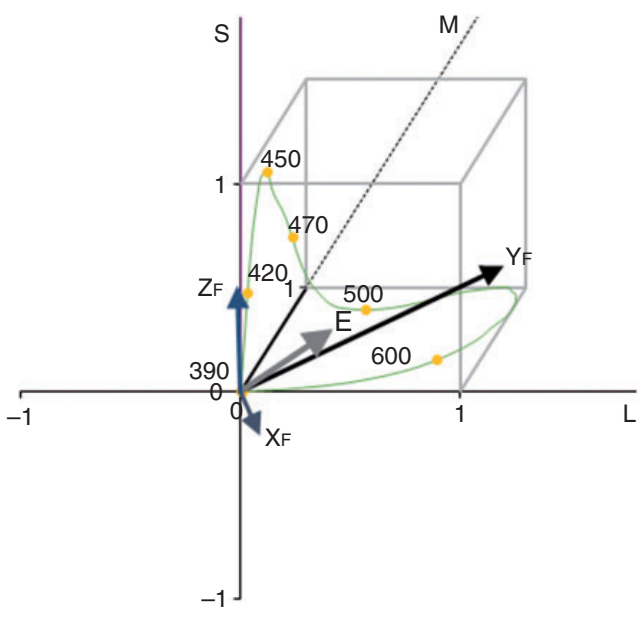

Figure 8. Illustration of the principles guiding the development of the cone-fundamental-based chromaticity diagram and the choice of the $X_{F}, Y_{F}, Z_{F}$ primaries. The monochromatic stimuli are represented in the LMS cone fundamental space where the cone fundamentals are scaled to unit-peak values. The selected wavelengths are given in $\mathrm{nm}$. E represents the equi-energy white.

3) The cone-fundamental-based tristimulus values of the equi-energy spectrum $\mathbf{E}$ are equal.

A few additional constraints are obeyed. 


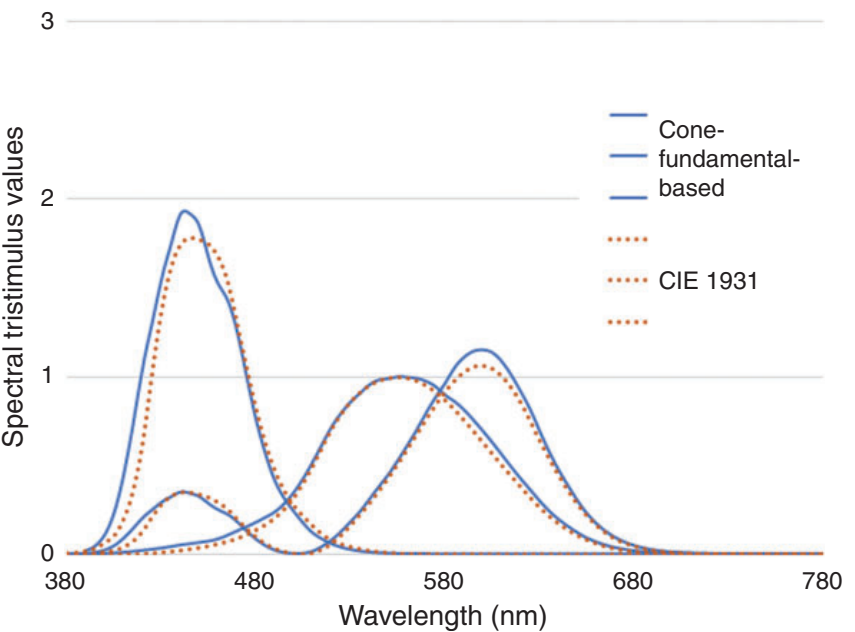

Figure 9. Comparison of the cone-fundamental-based spectral tristimulus values $\bar{x}_{F}(\lambda), \bar{y}_{F}(\lambda)$ and $\bar{z}_{F}(\lambda)$ with the CIE 1931 standard colorimetric observer.

4) The values of $z_{\mathrm{F}}(\lambda)$ are proportional to the values of the S-cone fundamental.

5) The minimum value of $x_{\mathrm{F}}$ is the same as in the $(x, y)$ chromaticity diagram. ${ }^{15}$

To be close to current practice in colorimetry, the overall difference between the cone-fundamental-based spectral chromaticity coordinates and the corresponding spectral chromaticity coordinates in the $(x, y)$ chromaticity diagram of the CIE standard colorimetric system is minimized. In fact, it has been shown that minimizing the difference between chromaticity diagrams yields closer colorimetric specifications than minimizing the difference between colourmatching functions. ${ }^{16}$

Finally, $2^{\circ}$ and $10^{\circ}$ fundamental colorimetric observers are available. The conefundamental-based spectral tristimulus values show some difference with the CIE 1964 standard colorimetric observer and marked differences with the CIE 1931 standard colorimetric observer (Figure 9).

All numerical data are available in the CIE publications and on the Colour and Vision Research Laboratory website, ${ }^{17}$ the number of significant digits being sufficient to allow high precision colorimetric calculations.

\section{Conclusion and perspectives}

CIE Publication 170-1:2006 provides the scientific community with a set of cone fundamentals for the average colour normal observer, ranging in visual angle from $1^{\circ}$ to $10^{\circ}$. These cone fundamentals have been derived from the best set of colour-matching functions available. While the primary goal of CIE TC1-36 was to propose cone fundamentals, the final goal of the work has to be to derive chromaticity diagrams. In this paper, a fundamental link is established between photometry and colorimetry. Two chromaticity diagrams are proposed, one for fundamental research and the other for straightforward colorimetric purposes.

For several decades, the cone fundamental approach has successfully been exploited by the scientific community. In the future, specifying colour in the LMS space will offer novel opportunities for applications through the CIE divisions. Several questions to be addressed 
by CIE divisions could benefit from the cone fundamental framework: The variability of individual colour responses, building improved colour appearance models, the photometry of punctiform light sources, the colorimetry of solid-state light sources, interpreting colour differences at various viewing angles, the measurement of colour temperature, discussing colour rendering and colour rendition, measuring road and vehicle lighting, assessing the melanopsin contribution to visual responses and non-imaging visual functions and colour specification in enlarged gamut image displays. Ultimately, it is expected that the link that has been established between colorimetry and fundamental physiology will improve the understanding of colour, will be useful for education and will offer novel opportunities to solve problems of colour measurement and colour perception in everyday life and industry.

\section{Acknowledgements}

I would like to acknowledge the contribution of all members of CIE TC 1-36 to the debate, with a special mention for Pieter Walraven (who passed away in 2014) and Andrew Stockman and for Jan-Henrik Wold for valuable assistance in the writing of the Technical Committee report. I am grateful to Robert Sève, to the reviewers and to the editor for relevant comments.

\section{Declaration of conflicting interests}

The author declared no potential conflicts of interest with respect to the research, authorship, and/or publication of this article.

\section{Funding}

The author received no financial support for the research, authorship, and/or publication of this article.

\section{References}

1 Judd DB. Fundamental studies of color vision from 1860 to 1960. Proceedings of the National Academy of Sciences 1966; 55: 1313-1330.

2 Commission Internationale de l'Eclairage. CIE Publication 170-1-2006 Fundamental Chromaticity Diagram with Physiological Axes. Vienna: CIE, 2006. CIE Publication 170-2 (in press).

3 Stockman A, Sharpe LT. The spectral sensitivities of the middle- and long-wavelength-sensitive cones derived from measurements in observers of known genotype. Vision Research 2000; 40: 1711-1737.

4 Stiles WS, Burch JM. N.P.L. colour-matching investigation: Final report (1958). Optica Acta 1959; 6: 1-26.

5 MacLeod DIA, Boynton RM. Chromaticity diagram showing cone excitation by stimuli of equal luminance. Journal of the Optical Society of America 1979; 69: 1183.

6 Stockman A, Sharpe LT. Cone spectral sensitivities and color matching. In: Gegenfurtner KR, Sharpe LT. (eds) Color Vision: From Genes to Perception. Cambridge: Cambridge University Press, 1999: pp. 53-87.

7 Stockman A. Physiologically-based colour matching functions: Commission Internationale de l'Eclairage. Proceedings of CIE 2012 "Lighting Quality and Energy Efficiency", Hangzhou, China, September 19-21: 2012: pp. 49-56.

8 Stiles WS, Burch JM. Interim report to the Commission Internationale de l'Eclairage, Zurich, 1955, on the National Physical Laboratory's investigation of colour-matching (1955). Optica Acta 1955; 2: 168-181.

9 Viénot F, Walraven P. Colour-matching functions, physiological basis. In: Schanda J. (ed) Colorimetry: Understanding the CIE System. Vienna: CIE and Hoboken, NJ: Wiley-Interscience, 2007: pp. 219-243.

10 Commission Internationale de l'Eclairage. CIE Publication 86-1990 2० Spectral Luminous Efficiency Function for Photopic Vision. Vienna: CIE, 1990.

11 Sharpe LT, Stockman A, Jägle H, Nathans J. Opsin genes, cone photopigments, color vision, 
and color blindness. In: Gegenfurtner KR, Sharpe LT. (eds) Color Vision: From Genes to Perception. Cambridge: Cambridge University Press, 1999: pp. 3-52.

12 Sharpe LT, Stockman A, Jagla W, Jagle H. A luminous efficiency function, $V^{*}(\lambda)$, for daylight adaptation. Journal of Vision 2005; 5: 3.

13 Sharpe LT, Stockman A, Jagla W, Jägle H. A luminous efficiency function, VD65* $(\lambda)$, for daylight adaptation: A correction. Color Research and Application 2011; 36: 42-46.

14 Wold JH, Valberg A. General method for deriving an XYZ tristimulus space exemplified by use of the Stiles-Burch $19552^{\circ}$ color matching data. Journal of the Optical Society of America A 1999; 16: 2845.

15 Commission Internationale de l'Eclairage. CIE Publication 15-2004. Colorimetry. 3rd Edition, Vienna: CIE, 2004.

16 Wold JH, Valberg. The derivation of XYZ tristimulus spaces: A comparison of two alternative methods. Color Research and Application 2001; 26: S222-S224.

17 Colour and Vision Research Laboratory and database, Colour \& Vision Research laboratory and database, Institute of Ophthalmology, University College London. Retrieved from http://www.cvrl.org/ (accessed 17 December 2015). 